\title{
outlook
}

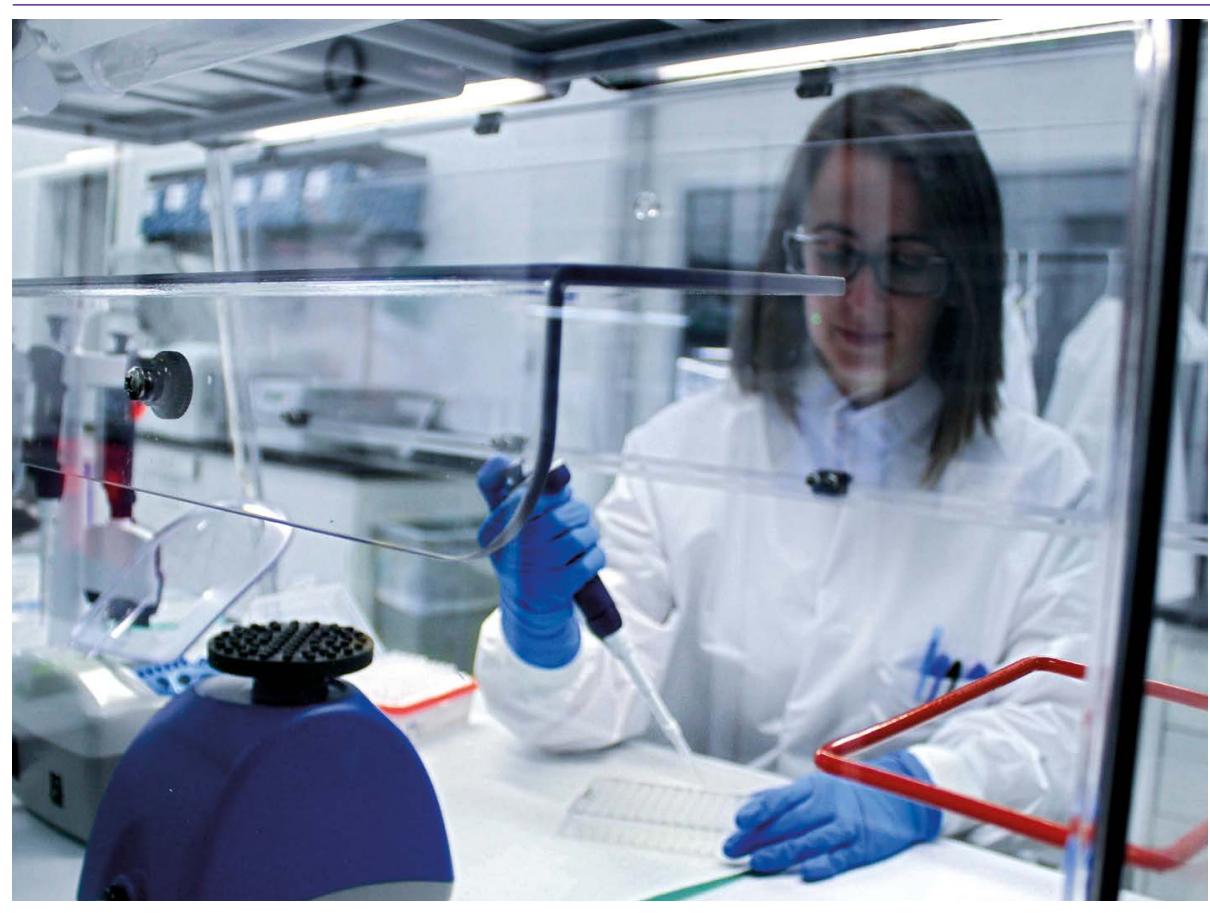

A scientist prepares samples for the UNITY blood test, which can screen for cystic fibrosis.

\section{The sooner the better}

\section{To safeguard organs, therapies must tackle cystic fibrosis in the womb. By Elie Dolgin}

W hen ivacaftor hit the market in 2012, becoming the first medicine to treat the underlying cause of cystic fibrosis (see page S2), it was initially available only for people aged six years and older. Trial data from younger children helped to lower the age limit to two. Then one. And in 2019, regulatory authorities approved the drug for infants as young as six months old who carry certain mutations in cystic fibrosis transmembrane conductance regulator (CFTR), the gene responsible for the disease.

Doctors are already evaluating the safety and efficacy of giving ivacaftor to newborn babies diagnosed with cystic fibrosis. And it's conceivable that they could begin treatment even earlier, perhaps months before birth - thanks in part to the advent of a simple blood test for pregnant women that can diagnose cystic fibrosis in utero.

Many babies born with cystic fibrosis already have damage to their organs that can lead to diabetes, gastrointestinal issues and male infertility. “The reality is that there's a lot of tissue damage that cannot be corrected," says Graça Almeida-Porada, a fetal-therapy researcher at the Wake Forest Institute for Regenerative Medicine in Winston-Salem, North Carolina.

But animal studies suggest that prenatal treatment with drugs such as ivacaftor might prevent organ damage. And gene therapy could even be used to fix the faulty CFTR gene before birth. "Correcting this in utero would be the best plan," says Craig Hodges, a mouse geneticist at Case Western Reserve University in Cleveland, Ohio, who has studied the effects of tackling the disease at different gestational time points in mouse development. "The earlier you can get in, the better."

\section{Fetal fix}

The idea of prenatal intervention has a long and storied history in the cystic fibrosis field. More than 20 years ago, a team led by neonatologist Janet Larson at the Ochsner Medical Foundation in New Orleans, Louisiana, described the first application of in utero gene therapy in mouse models of the disease ${ }^{1}$. After that 1997 report, Larson recalls, "I had mothers contacting me about in utero gene transfer, asking me to do it for their babies." Most cysticfibrosis researchers, however, were fairly sceptical of the study results.

Larson and her colleagues had injected viruses encoding working copies of the CFTR gene into the uterine sac surrounding fetal mice. These mice were fated to develop life-threatening bowel obstructions owing to defects in CFTR, which encodes a cellsurface protein that is needed for maintaining the proper balance of salt and water in organ systems throughout the body. But after swallowing virus-filled amniotic fluid during the second trimester of pregnancy, the mice developed normally and survived for more than a year into adulthood with no discernible intestinal problems, Larson's team reported.

Most surprisingly - and controversially the disease fix came about from just a few days of transient gene expression in the fetal gut. No traces of CFTR protein activity were detectable after birth. Contrary to the prevailing view that continuous functioning of CFTR is needed to prevent disease symptoms in adults, the results seemed to indicate that there was a critical interval during fetal development when switching on the gene, even briefly, could lead to the correct patterning of cells in the intestines, airways and elsewhere.

Larson's team replicated the findings in fetal rats and monkeys ${ }^{2,3}$. "The next step would have been clinical trials," she says. Then came the death, in 1999, of an 18-year-old volunteer in an experimental gene-therapy study (unrelated to cystic fibrosis). This set the entire field of gene therapy back many years. Funding dried up. The research stalled. The final death knell for Larson's unconventional method came in 2008, when two prominent UK groups published back-to-back studies in which they each tried and failed to reproduce her initial mouse data ${ }^{4,5}$.

Those reports "killed" any scientific momentum she had left, says Larson, who now runs a perinatal medicine clinic in Ephrata, Pennsylvania. "After those articles, it was difficult to continue." And across the cystic fibrosis research community, scientists mostly abandoned their pursuit of fetal gene therapies - until a few years ago, when older gene-transfer approaches gave way to newer gene-editing tools.

With the advent of technologies such as CRISPR, it has now become possible to precisely correct CFTR mutations in cells, rather than simply transfer extra normal copies of the gene, as Larson did with the virus-laden amniotic-fluid doses. This greater level of control has brought renewed interest in the 
long-sought goal of fixing the disease as early as possible.

Last year, for example, a team from the University of Pennsylvania (UPenn) in Philadelphia remedied a lethal lung disease in fetal mice by introducing CRISPR gene editors into the amniotic fluid late in pregnancy ${ }^{6}$. Without the treatment, the young pups struggled for air after birth; no mice lived beyond six hours. With the gene fix, 5 of the 87 mice tested were healthy, with no signs of laboured breathing.

The researchers focused their efforts on a lung disorder called surfactant deficiency, not cystic fibrosis. But given the overlap between the two diseases - both can cause respiratory failure and both involve genetic defects in airway cells - lead author William Peranteau is hopeful that the strategy will work for cystic fibrosis as well. "Our initial study of in utero gene editing should be translatable to other genetic lung diseases," says Peranteau, a fetal surgeon at the Children's Hospital of Philadelphia.

Elsewhere, researchers at Yale University in New Haven, Connecticut, have used a new gene-editing platform to replace faulty versions of CFTR with working copies in fetal mice. The platform involves biodegradable nanoparticles carrying a DNA correction sequence along with strands of synthetic genetic material that trigger the cell's natural DNA repair mechanisms.

\section{Precise edits}

The research builds on two previous studies from a team co-led by paediatric pulmonologist Marie Egan, biomedical engineer Mark Saltzman and geneticist Peter Glazer. In 2015, these Yale scientists showed that their sitespecific editing technology could fix the most common CFTR mutation, known as F508del, in human lung cells and adult mice ${ }^{7}$. They then adapted the technique for in utero treatment, providing proof-of-concept for the therapeutic approach in a mouse model of the blood disorder $\beta$-thalassaemia ${ }^{8}$. And most recently, in as-yet-unpublished work, the Yale team has achieved prenatal repair of the CFTR gene.

"We do have site-specific gene correction that's long term in these mice," says Adele Ricciardi, a former graduate student at Yale (now in surgical training at UPenn) who presented the findings at various scientific conferences in 2019. The results, she says, show that "treating at the time of pathogenesis in utero could be a compelling strategy".

Of course, prenatal treatment is only relevant if there is prenatal diagnosis, and fetuses are rarely tested for cystic fibrosis. Only when both parents are known carriers of CFTR mutations, or when second-trimester ultrasound scans reveal bowel irregularities, do doctors typically recommend fetal testing.

What's more, not every country offers routine screening to pregnant women to see whether they carry a faulty CFTR gene. Among women who do test positive for cystic fibrosis-associated mutations, their partners often fail to pursue the follow-up screening needed to clarify the couple's reproductive risk.

And even when expectant couples know they are both carriers (meaning there is a $25 \%$ chance each parent has passed on the mutated gene) or they see brighter-than-usual bowels on the ultrasound (a sign of cystic fibrosis in about $3 \%$ of cases), many opt not to test their babies in utero because doing so usually requires removing cells from the amniotic fluid or placenta, an invasive procedure that carries a risk of fetal injury and miscarriage.

Now, however, a simple blood test, similar to the one used to screen for Down's syndrome and other chromosomal differences in utero, can reveal single-gene disorders as early as ten weeks into a pregnancy. The UNITY test, from diagnostics company BillionToOne in Menlo Park, California, uses a molecular counting method to detect fetal DNA circulating in the mother's bloodstream?. From a single tube of maternal blood, it can

\section{"It really shows that there is this in utero period when it is really important to have CFTR function."}

accurately identify whether the baby has inherited any of five disorders, including cystic fibrosis.

"With just one test, we tell parents exactly what they want to know," says Oguzhan Atay, co-founder and chief executive of BillionToOne, which began selling its UNITY test last year. Because of the simplified workflow, "I think it will become the norm," he says. "This is the best way to identify these babies during pregnancy."

Notably, the non-invasive blood test determines not only whether the fetus has cystic fibrosis, but also the exact nature of the mutations responsible for the disease. That information will be important for deciding who should undergo in utero gene editing, because the benefits of the therapy might outweigh the risks only for those fetuses that carry the most severe mutations in the CFTR gene. "We have to be cautious and to know which mutations to target," Almeida-Porada says.

Identifying the mutation is also essential for selecting drug therapies, because CFTR-modulating agents such as ivacaftor only work against specific types of CFTR mutation. Knowing the fetal genotype, says Edward Nash, a respiratory physician at the University Hospitals Birmingham NHS Foundation Trust, UK, "would potentially enable the mother to take CFTR modulators to prevent the baby being born with complications such as pancreatic insufficiency and male infertility".

\section{The leap to humans}

Earlier this year, Nash and his colleagues published an international survey looking at the safety of cystic fibrosis drugs taken during pregnancy ${ }^{10}$. All the cases they looked at involved women with cystic fibrosis who were taking the drugs for themselves, not their unaffected fetuses, so the survey could not gauge the efficacy of in utero drug treatment. But it showed that fetal exposure to CFTR modulators does not seem to cause any major complications. And if the results from a ferret model of cystic fibrosis translate to people, there's reason to hope that the drugs could prevent multi-organ damage in disease-affected fetuses as well.

Like many humans, ferrets with CFTR mutations are born with intestinal, pancreatic and male reproductive abnormalities. When their mothers start taking ivacaftor at the start of the third trimester of pregnancy, however, those organs are protected, and sustained administration of the drug after birth helps to keep the disease symptoms at bay ${ }^{11}$.

"It really shows that there is this in utero period when it is really important to have CFTR function," says John Engelhardt, a cystic fibrosis researcher at University of Iowa in Iowa City, who reported the findings last year. Human studies will be needed to validate the result, but with ivacaftor approved for six-montholds and already being given to newborns in experimental trials, Engelhardt anticipates a day, soon, when the drug will be used for fetal treatment. “It's probably coming," he says. "It's a natural progression."

Elie Dolgin is a science journalist in Somerville, Massachusetts.

\footnotetext{
. Larson, J. E., Morrow, S. L., Happel, L., Sharp, J. F. \& Cohen, J. C. Lancet 349, 619-620 (1997).

2. Morrow, S. L. et al. Mol. Genet. Metab. 65, 203-212 (1998).

3. Larson, J. E. et al. Mol. Ther. 2, 631-639 (2000).

4. Davies, L. A. et al. Mol. Ther. 16, 812-818 (2008)

Buckley, S. M. K. et al. Mol. Ther. 16, 819-824 (2008).

. Alapati, D. et al. Sci. Transl. Med. 11, eaav8375 (2019).

McNeer, N. A. et al. Nature Commun. 6, 6952 (2015)

Ricciardi, A. S. et al. Nature Commun. 9, 2481 (2018)

9. Tsao, D. S. et al. Sci. Rep. 9, 14382 (2019).

10. Nash, E. F., Middleton, P. G. \& Taylor-Cousar, J. L. J. Cyst.

Fibros. 19, 521-526 (2020).

11. Sun, X. et al. Sci. Transl. Med. 11, eaau7531 (2019).
} 